\section{Carcinógeno en la orina de recién nacidos de madres que fuman}

En la primera orina de bebés cuyas madres fuman durante el embarazo se ha encontrado la nitrosamina cancerígena 4-(metilnitrosamino)-1-(3piridil)-1 butanona (NNK). Stephen Hecht, científico afiliado al centro de estudios sobre el cáncer de la Universidad de Minnesota, Estados Unidos de América, condujo los estudios que llevaron a este descubrimiento. La NNK es uno de los cancerígenos más potentes derivados del tabaco y los hallazgos del doctor Hecht sugieren que la sustancia no solo es recibida sino metabolizada por el feto.

Se informó de esta novedad a la reunión de la Sociedad Americana de Químicos, que se celebró en Boston en agosto. Para el estudio, se analizaron a ciegas mediante cromatografía de gas muestras de la primera orina de 48 recién nacidos. Los metabolitos de NNK se detectaron en 22 de las 31 muestras de bebés cuyas madres habían fumado cuando estaban embarazadas. No se encontraron metabolitos de esa clase en los bebés de las madres que no fumaban. La concentración de productos de NNK en los recién nacidos corresponde a $10 \%$ de los que se encuentran en los adultos fumadores. No obstante, constituye una exposición considerable para el feto, ya que la madre suele mantener el hábito de fumar durante todo el embarazo. Los resultados del estudio se consideran concluyentes, pues las concentraciones de metabolitos encontradas fueron muy altas en proporción al tamaño de los bebés. Lo más probable es que los bebés excreten estas sustancias en el fluido amniótico, donde recircula constantemente.

Por supuesto, es bien conocido que el hábito de fumar en una embarazada se asocia con bajo peso al nacer, desarrollo subnormal y problemas del oído que pueden requerir cirugía. Habiendo datos que indican que $60 \%$ de las mujeres que fuman mantienen el hábito durante la gestación, esta información debe usarse para fortalecer los mensajes al público sobre los efectos del hábito de fumar en las embarazadas. (Wise J. Carcinogen in tobacco smoke can be passed to fetus. Br Med J 1998;317:555.)

\section{Un mejor detector de vinchucas para uso doméstico}

La zona endémica de la enfermedad de Chagas en la Argentina es de gran extensión y, a pesar del tratamiento masivo de las viviendas contra Triatoma infestans, la incidencia de reinfestación es muy alta. Por ello es importante contar, no solo con estrategias de control eficientes, sino con instrumentos útiles y económicos que las complementen. El "sensor María" (SM) se diseñó con ese fin en los años ochenta y se ensayó con buenos resultados en la provincia de Santa Fe. Este detector pasivo de las vinchucas -insectos hematófagos vectores de la enfermedad de Chagas - consiste en una caja de cartulina de $22 \mathrm{~cm}$ de altura, $40 \mathrm{~cm}$ de ancho y $2 \mathrm{~cm}$ de profundidad con orificios de entrada para los insectos.

Sin embargo, debido al deterioro de la estructura de atención primaria de salud, en 1991 el uso de los SM estuvo a cargo de los propios pobladores en dos localidades del área endémica. En esa oportunidad se encontraron varias dificultades debidas probablemente a la manipulación inhábil de esas personas. La cartulina era de grosor muy fino y al retirarlas de las paredes para revisarlas, las cajas se rompían fácilmente. Al rearmarlas se aplastaba la base, lo cual ocluía los orificios de entrada y a menudo la parte interior era colocada al revés con el mismo efecto. Para solucionar esas dificultades, se diseñó un modelo diferente, denominado "detector Santa Fe" (DSF). Esta vez se construyó de una sola pieza de cartulina, para simplificar el armado y garantizar el rearmado correcto. La cartulina es más espesa y fuerte que la de las cajas anteriores, lo que redujo su ruptura. También se mejoró el diseño y la colocación de las aberturas en los extremos de las canaletas para realizar el plegado con mayor facilidad y brindar más oscuridad al interior del detector. Para comparar la eficacia de los dos detectores, se llevaron a cabo dos evaluaciones en un área de alta endemicidad, el distrito de Villa Minetti en el departamento 9 de julio. En esta ocasión se seleccionaron 63 viviendas y se probaron en total 172 detectores de ambos modelos. Se colocaron un SM y un DSF por dormitorio, ambos en la cabecera de la misma cama a $150 \mathrm{~cm}$ del piso y separados por una distancia de $30 \mathrm{~cm}$. Las viviendas positivas se trataron inmediatamente después de las evaluaciones, a los 45 y 90 días. En 22 (35\%) de las 63 viviendas se encontraron detectores con vinchucas vivas o indicios de su presencia (exuvias, huevos o rastros fecales y de orina). Siete viviendas fueron positivas con ambos modelos de detector, otra solo con el SM y 14 con el DSF. La sensibilidad mostrada por el DSF fue notablemente superior a la del SM. Además, el 
costo por unidad del DSF fue de US $\$ 1,00$ y el de $\mathrm{SM}$ de $\$ 3,00$. De manera que el nuevo modelo de detector de vinchucas es más sensible y más barato que el anterior, ventaja de gran importancia en países donde los programas de salud tienen presupuestos muy limitados. (Candioti C, Paulone I. Detector de vinchucas Santa Fe: un diseño de alta sensibilidad y bajo costo. Medicina (Buenos Aires) 1997;57:433-436.)

\section{Resistencia a las drogas antituberculosas en todo el mundo}

La resistencia de Mycobacterium tuberculosis a los antibióticos se debe a la ampliación artificial de mutaciones genéticas espontáneas. El tratamiento con un solo medicamento debido a irregularidad del aprovisionamiento, prescripción inadecuada o mala observancia del tratamiento suprime el desarrollo de cepas susceptibles al medicamento a la vez que permite que se multipliquen las resistentes. Este fenómeno se denomina resistencia adquirida. La transmisión subsecuente de cepas resistentes de un caso infeccioso a otras personas produce enfermedad farmacorresistente por naturaleza, fenómeno que se conoce por el nombre de resistencia primaria. Los brotes importantes de tuberculosis multirresistente (TB-MR) - es decir, resistente a múltiples medicamentos- que han surgido en pacientes infectados con VIH en los Estados Unidos de América y Europa han atraído la atención internacional hacia la emergencia de cepas de $M$. tuberculosis resistentes a los antimicobacterianos. La TB-MR, que se define como resistencia a los dos medicamentos antituberculosos más importantes, la isoniazida (INH) y la rifampicina (RMP), representa una grave amenaza para el control de la tuberculosis. Los pacientes infectados con cepas de TB-MR son muy difíciles de curar y necesitan tratamientos mucho más tóxicos y costosos.

Se desconoce cuán extendido está el problema. Las tasas de resistencia parecen haber aumentado en algunos entornos, pero obstáculos metodológicos han impedido evaluar correctamente ese incremento. A principios de 1994, el Programa Mundial de Lucha contra la Tuberculosis (OMS) y la Unión Internacional contra la Tuberculosis y las Enfermedades Respiratorias establecieron un grupo de trabajo conjunto para vigilar la resistencia a los medicamentos en el ámbito mundial. Los objetivos del proyecto eran medir la prevalencia de la resistencia antituberculosa por medio de métodos normalizados y estudiar la correlación entre el nivel de farmacorresistencia y las políticas nacionales de tratamiento. Como primer paso, expertos internacionales establecieron definiciones y directrices de vigilancia (revisadas en 1997) que se centraban en tres principios: vigilancia basada en una muestra representativa de pacientes de TB en cada país; diferenciación de los casos de resistencia adquirida y primaria, y garantía del buen funcionamiento de los laboratorios. En segundo lugar se estableció una red mundial de laboratorios de referencia supranacionales, que actualmente cuenta con 24 de ellos. Como tercer paso, se organizó un grupo de trabajo bajo la dirección de la OMS con representantes de programas nacionales de TB e instituciones de investigación de más de 50 países para poner en práctica proyectos de vigilancia. La primera fase del proyecto obtuvo resultados de 35 países de cinco continentes, en los que se vigilaron o encuestaron 50000 casos de TB de zonas que representan $20 \%$ de la población mundial. En los estudios llevados a cabo se evaluó la resistencia a INH y RMP, y también a etambutol (EMB) y estreptomicina (SM), con una concordancia general de $96 \%$ entre laboratorios de diversos países.

Los resultados indican que hay varios lugares donde se requiere intervención urgente para salvar los programas de control de la TB: Estonia, Latvia y la Federación de Rusia en Europa; Argentina y la República Dominicana en las Américas y Côte d'Ivoire en África. La calidad general de las actividades de lucha antituberculosa y el uso de tratamientos estandarizados de corta duración se correlacionan fuertemente con una menor proporción de farmacorresistencia. La alta prevalencia de TB-MR revela una anarquía terapéutica. La mitad de los lugares con el peor control de TB tenían resistencia primaria de más de $2 \%$ en contraste con un quinto de aquellos con control de calidad mediana y ninguno de los países con los mejores programas de lucha. La prevalencia de TB-MR primaria es un buen indicador de la calidad de los programas.

De ello se derivan las siguientes recomendaciones: 1) Mantener como recurso mundial la red de laboratorios de referencia supranacionales y repetir la encuesta de 35 países alrededor del año 2000 para observar la tendencia diacrónica de la TB-MR. 2) Para evaluar correctamente los niveles de TB-MR en países grandes como China, India y la Federación de Rusia, es preciso expandir la vigilancia a zonas no cubiertas en la primera fase del proyecto. 3) Analizar, en las encuestas futuras, datos individuales sobre edad, país de nacimiento, coinfección TB-VIH y contribución del sector privado a la resistencia de los medicamentos. Además, debe establecerse la estrategia de tratamiento bajo observación directa (TOD) en los países que no la tienen. La experiencia muestra que las tasas de resistencia bajan en los países que emplean la TOD.

Si bien no se abordó el tema de los regímenes de tratamiento, no se considera necesario hacer cambios en los de primera línea recomendados por 
la OMS y la Unión. Para el tratamiento de la TB resistente, incluida la TB-MR, el lector puede solicitar el documento inédito del Programa Mundial de Tuberculosis de la OMS "Guidelines for the management of drug-resistant tuberculosis" (WHO/TB/ 96.210). Por otra parte, los investigadores deben evaluar la transmisibilidad y virulencia clínica de la enfermedad causada por cepas multirresistentes frente a la causada por cepas susceptibles y definir el impacto de la TB-MR en los resultados del tratamiento ofrecido por los programas en los países en desarrollo. Por último, se insta a las compañías farmacéuticas a desarrollar nuevos medicamentos antituberculosos. (Organización Mundial de la Salud. Antituberculosis drug resistance worldwide. Wkly Epidemiol Rec 1998;73:249-254.)

\section{Intervalos intergenésicos cortos en poblaciones de bajos recursos}

La noción tradicional de que los intervalos cortos entre embarazos son un factor de riesgo de resultados precarios y en particular de mortalidad infantil en los países en desarrollo se sustenta en las hipótesis de agotamiento de la madre, estrés posparto y otras. La hipótesis de agotamiento materno sugiere que es esencial que transcurra uno o más años entre el nacimiento de un niño y la concepción de otro para permitir que se restauren los recursos nutricionales de la madre necesarios para llevar a término con éxito el embarazo. Sin embargo, algunos investigadores han planteado dudas sobre si el síndrome de agotamiento materno se debe a los intervalos cortos o a nutrición inadecuada. En cambio, la hipótesis del estrés posparto se fundamenta en que el cuidado de un niño pequeño produce estrés físico y emocional que interfiere con el crecimiento del feto o la duración del embarazo subsecuente. Dados los resultados diversos y discrepantes de muchos estudios anteriores, se realizó un estudio para determinar si la duración del intervalo entre embarazos se asociaba con nacimientos pretérmino o retardo del crecimiento intrauterino en una población de bajos recursos mayoritariamente de raza negra.

La población constó de 4400 mujeres que habían recibido atención prenatal en clínicas públicas y habían tenido dos nacimientos consecutivos de un solo niño entre 1980 y 1990. El intervalo intergenésico se midió en semanas desde la fecha del parto del primer niño y la del segundo menos la edad gestacional de este último. La mayor parte de las mujeres eran jóvenes -3 145 tenían de 20 a 29 años de edad - y pobres. La mayoría (90\%) recibían asistencia social. Más de la mitad iniciaron su atención prenatal en el segundo o tercer trimestre de gestación.
De esta población, $2,4 \%$ tuvieron un intervalo de menos de 13 semanas entre embarazos; 7,5\%, de 13 a 25 semanas; $17,3 \%$, de 26 a 51 semanas; $26,8 \%$, de 52 a 103 semanas; y 46\%, de 104 semanas o más. Los intervalos intergenésicos fueron de menos de un año en más de una cuarta parte de las mujeres y se asociaron positivamente con la edad. El grupo más joven, de menores de 20 años, constituyó el porcentaje más alto $(19,4 \%)$ de las que tuvieron intervalos muy cortos (menos de 26 semanas). Aun entre el grupo de 20 a 29 años de edad, la mitad tuvo un segundo hijo en menos de 2 años. Solo en las de 30 o más años (12\% de la muestra) hubo una mayoría $(57,6 \%)$ que tuvieron su segundo hijo después de un intervalo de 2 o más años. Las mujeres de raza blanca tuvieron intervalos más cortos que las de otras razas. Mientras más corto el intervalo, más tarde se inició la atención médica en el segundo embarazo. El porcentaje de nacimientos pretérmino se relacionó directamente con intervalos intergenésicos más cortos, pero solamente en las mujeres que no habían tenido nacimientos pretérmino anteriormente. La asociación entre el intervalo y los nacimientos pretérmino se mantuvo aun cuando se controlaron otros factores asociados con los nacimientos pretérmino. No se encontró ninguna relación significativa entre el retardo del crecimiento intrauterino y el intervalo entre embarazos.

Todas las mujeres de edad fecunda deben recibir asesoramiento sobre el perjuicio potencial que representan los intervalos cortos entre embarazos. (Klerman LV, Cliver SP, Goldenberg RL. The impact of short interpregnancy intervals on pregnancy outcomes in a low-income population. Am J Public Health 1998;88:1182-1185.)

\section{Cambios en la mortalidad por cardiopatías en los Estados Unidos}

Se estima que, de los 4,8 millones de residentes de los Estados Unidos que padecen insuficiencia cardíaca, 70\% tienen 60 años o más de edad. Durante los últimos 10 años, entre los beneficiarios del Medicare $^{1}$ ha aumentado el número de hospitalizaciones por esa causa. A pesar de que la tasa de letalidad por insuficiencia cardíaca es alta, es de esperar que ese número siga creciendo a medida que la población envejece. Un informe de los Centros para el Control y la Prevención de Enfermedades (CDC) manifiesta las tendencias del país en insuficiencia cardíaca durante el período de 1980 a 1995.

\footnotetext{
Programa social de seguro de enfermedad para personas de 65 o más años, personas que hayan recibido desembolsos por discapacidad durante 2 o más años y ciertos trabajadores y sus dependientes que necesiten trasplantes renales o diálisis.
} 
En los Estados Unidos, las estadísticas de mortalidad se basan en la información consignada en los certificados de defunción registrados en las oficinas de estadísticas vitales y compilados por los CDC. Los datos sobre causas de muerte se refieren a las causas subyacentes registradas por el médico de cabecera o el médico forense en la manera prescrita por la OMS y aprobada por los CDC. Las tasas de mortalidad de la población estadounidense se calcularon mediante estimaciones de la Oficina de Censos. Las muertes por insuficiencia cardíaca se definieron como las de causa subyacente registrada con el código 428 de la Clasificación Internacional de Enfermedades (CIE-9). Esta categoría incluye la insuficiencia cardíaca congestiva, ventricular izquierda y no especificada.

En los 15 años que abarca el informe, las muertes por insuficiencia cardíaca aumentaron de 27415 a 46 484; en 1995, aproximadamente 43600 (94\%) de ellas fueron de personas de 65 o más años de edad. La tasa general, que era de 10,3 por 100000 en 1980, llegó a 11,7 en 1995. Las tasas de mortalidad por insuficiencia cardíaca fueron directamente proporcionales a la edad. Por ejemplo, en 1995, la tasa específica por edad del grupo de 85 o más años era 633.5; la del grupo de 75 a $84,130,8$ y la del grupo de 65 a 74, 32,2. A partir de 1988 hubo una redución de las tasas de mortalidad por esa causa en ambos sexos y en todos los grupos raciales. Además, la reducción anual más importante ocurrió en hombres de raza negra ( $3 \%$ ) seguida de la de mujeres de raza negra $(2,2 \%)$, hombres de raza blanca $(1,7 \%)$ y mujeres de raza blanca $(0,5 \%)$. En consecuencia, la brecha entre las dos razas disminuyó y la razón blanco: negro descendió de 1.3:1 a 1.1:1 en los hombres. Entre las mujeres descendió de 1.4:1 a 1.1:1.

En 1995 las tasas de IC ajustadas por edad de todos los grupos variaron mucho de un estado a otro. Los extremos fueron Nueva Hampshire $(3,4)$ y Misisipí $(29,7)$. En las personas mayores de 65 años, esas tasas oscilaron entre 30,7 (Nueva Hampshire) y 255,6 (Alabama). La disminución registrada quiere decir o mejor supervivencia o diagnóstico equivocado de la causa subyacente de muerte en las personas mayores con IC. Este trastorno muchas veces se registra como causa contribuyente y no directa de muerte en los certificados de defunción. Aunque en realidad haya declinado la IC, deben continuarse los esfuerzos clínicos y de salud pública para enseñar a la gente a prevenir la hipertensión y el infarto agudo de miocardio, los dos factores prevenibles principales asociados con la IC. (Centers for Disease Control and Prevention. Changes in mortality from heart failure-United States, 1980-1995. JAMA 1988; 280(10):874-875.)

\section{Lagunas en la investigación de resistencia a los antibióticos}

El uso de las fluoroquinolonas para tratar al ganado pecuario puede contribuir a aumentar la resistencia de bacterias como Campylobacter y Salmonella que se transmiten a los seres humanos por medio de los alimentos. Las fluoroquinolonas son importantes en el tratamiento de infecciones invasoras por Salmonella y Campylobacter y el aumento de la resistencia de esas bacterias debe contemplarse con preocupación. Si bien hasta el momento no se han documentado extensamente los efectos en la salud humana del uso de estos antibióticos en el ganado, hay razones para considerar que puede haber consecuencias deletéreas para la salud humana si la resistencia aumenta y se dispersa. El doctor David Heymann, Director de la División de Vigilancia y Control de Enfermedades Emergentes y Otras Enfermedades Transmisibles, OMS, advierte que es necesario investigar y reunir datos sobre este posible problema.

Desde que se empezaron a emplear las fluoroquinolonas en varios países, en los animales comestibles se han encontrado salmonelas de susceptibilidad reducida a esos antibióticos. También han surgido cepas resistentes de Campylobacter. Se ha observado también que con el uso de fluoroquinolonas en humanos, han surgido cepas resistentes de microorganismos patógenos, lo que ha limitado los beneficios terapéuticos de estos antibióticos contra enfermedades importantes como la gonorrea y la fiebre tifoidea.

Con objeto de conocer el impacto médico del uso de quinolonas en animales, la OMS convocó una reunión de 60 expertos en salud humana y veterinaria, la cual se celebró en Ginebra en junio de 1998. En ella se acordó que en la investigación futura debe hacerse hincapié en determinar el alcance del uso de quinolonas en medicina no humana, mejorar el conocimiento epidemiológico de cómo se desarrolla, crece y se transmite la resistencia a antibióticos tanto en humanos como en animales, idear tecnologías de vigilancia específicamente para conseguir los datos que faltan, determinar los mecanismos y grados de resistencia a las quinolonas de organismos patógenos zoonóticos importantes y lo que significa esa resistencia en función de la salud humana, formular estrategias para un uso prudente que aproveche al máximo su efecto terapéutico en animales y minimice el desarrollo de la resistencia y buscar alternativas tales como vacunas en lugar de agentes antimicrobianos para la prevención de enfermedad en los animales. (Organización Mundial de la Salud. Major gaps in research on antibiotic resistance needs filling. Comunicado de prensa WHO/46, 9 de junio de 1998.) 